\title{
Immunization with Streptococcus suis bacterin plus recombinant Sao protein in sows conveys passive immunity to their piglets
}

Kai-Jen Hsueh ${ }^{1,2}$, Li-Ting Cheng ${ }^{2}$, Jai-Wei Lee ${ }^{3}$, Yao-Chi Chung ${ }^{2}$, Wen-Bin Chung ${ }^{1 *}$ and Chun-Yen Chu ${ }^{2^{*}}$

\begin{abstract}
Background: Streptococcus suis (S. suis) causes arthritis, meningitis, septicemia, and sudden death in pigs and is also an zoonotic agent for humans. The present study demonstrated that immunization with recombinant Sao- $L$ (surface antigen one-L, rSao-L) protein from a strain of S. suis serotype 2 in pigs was able to increase cross-serotype protection against $S$. suis serotype 1 and 2 challenge. Since weaning piglets are more susceptible to $S$. suis infections due to the stresses associated with weaning, prepartum immunization in sows may convey passive immunity to piglets and provide protection.
\end{abstract}

Results: Pregnant sows were immunized with a vaccine containing inactivated S. suis serotype 2 plus rSao as the antigens. Blood samples were collected from their piglets after birth for analysis of antigen-specific antibody titers and levels of various cytokines. Results demonstrated that the titers of $\mathrm{S}$. suis and rSao-specific antibodies were significantly $(p<0.05)$ higher in the vaccinated piglets in comparison with that of piglets in the control group. The serum levels of interferon (IFN)- $\gamma$, interleukin (IL)-4, IL-6, and IL-12 were significantly $(p<0.05)$ increased in piglets born from vaccinated sows when compared to piglets from unvaccinated sows. In addition, piglets were challenged by heterologous and homologous S. suis. All piglets from unvaccinated sows developed severe symptoms of bacteremia, fever, anorexia, depression, and arthritis. On the other hand, piglets from vaccinated sows had significantly $(p<0.05)$ reduced clinical symptoms and lesion score (by 75 and 81\%).

Conclusions: Our results revealed that immunizing pregnant sows with the vaccine containing inactivated S. suis bacterin plus rSao as the antigens is able to enhance passive immunity against heterologous and homologous $S$. suis challenge in their piglets.

Keywords: Streptococcus suis, rSao, Vaccine, Passive immunity, Cytokine

\footnotetext{
* Correspondence: wbchung@mail.npust.edu.tw; cychu@mail.npust.edu.tw ${ }^{1}$ Department of Veterinary medicine, College of Veterinary Medicine, National Pingtung University of Science and Technology, Pingtung 91201, Taiwan ${ }^{2}$ Graduate Institute of Animal Vaccine Technology, College of Veterinary Medicine, National Pingtung University of Science and Technology, 1, Shuehfu Road, Neipu, Pingtung 91201, Taiwan

Full list of author information is available at the end of the article
} the Creative Commons license, and indicate if changes were made. The Creative Commons Public Domain Dedication waiver (http://creativecommons.org/publicdomain/zero/1.0/) applies to the data made available in this article, unless otherwise stated. 


\section{Background}

Streptococcus suis (S. suis) causes a spectrum of diseases, including arthritis, meningitis, septicaemia and sudden death, in pigs and is recognized as an important zoonotic agent for humans [1]. A total of 33 serotypes of S. suis, according to the structure of its capsular polysaccharides (CPS), have been identified [2]. Serotypes 1, 1/2, 2, 7, 9, 14 and 22 are more virulent than others and can be isolated worldwide, including the United States, Canada, Europe, and New Zealand [3]. In Taiwan, more than $80 \%$ of the clinical cases are S. suis positive [4], and most of them are infected with serotypes 1 and 2 [5]. The efficacy of many commercially available inactivated $S$. suis whole-cell vaccines left something to be desired due to the fact that the protection is only limited to homologous strains [6]. The CPS has been shown to play a pivotal role in the pathogenesis of S. suis, and that antibody against CPS (serotype 2 ) is essential for full protection from homologous challenge [7]. CPS is a poor immunogen due to its thymus-independent property and cannot induce sufficient immune response [8]. Study showed that coupling $S$. suis type 2 CPS to tetanus toxoid can induce $\mathrm{T}$ celldependent response, inducing IgM and IgGs for protection against challenges in mice and pigs $[9,10]$. In addition, immunization with certain virulence factors, such as suilysin, muramidase-released protein (MRP) and extracellular factor (EF), has been demonstrated to protect pigs from challenge with homologous or heterologous strains of $S$. suis. However, discrepant results were reported when different virulence factors are expressed by the prevalent $S$. suis strains [11]. Therefore, researching for a more immunogenic antigen that is commonly expressed by most $S$. suis strains is imperative.

Many surface proteins are involved in the pathogenesis of Gram-positive bacteria and have been shown to elicit strong immune responses [12-14]. Immunization with recombinant $S$. suis SsnA protein (surface-anchored DNA-nuclease) formulated with aluminum hydroxide was able to protect mice from S. suis infection [15]. A new surface protein of $S$. suis, Abpb (amylase-binding protein B) is recently identified and immunization with recombinant $\mathrm{Abpb}$ provided effective protection against S. suis [16]. In addition, a Lam protein (CDS0330) was expressed on the cell surface of S. suis 2 and was found to bind laminin in vitro. Immunization with recombinant CDS 0330 protein resulted in 50\% survival rate of mice from S. suis 2 infection [17]. S. suis, Sao (surface antigen one), a common surface protein containing an Leu-Pro-X-Thr-Gly (LPXTG) motif, mediates many virulence factors during the course of infection. Sao is highly conserved among $S$. suis strains and has become a potential antigen for the development of effective vaccines against S. suis [13]. Sao protein is encoded by three allelic variants of gene with different lengths, Sao-S
(1.5 kb), Sao-M (1.7 kb) and Sao-L (2.0 kb), respectively, and Sao-M is the most prevalent variant [14]. Immunization with rSao protein was able to elicit strong humoral antibody responses, decrease clinical signs and bacterial dissemination, increase survival rates, and confer cross-serotype protection in mouse and pig vaccination protocols [18], indicating that $\mathrm{rSao}$ is a suitable antigen for subunit vaccine development.

We previously demonstrated that immunization with recombinant Sao-L protein (rSao-L) from a strain of $S$. suis serotype 2 in pigs was able to increase antigenspecific antibody titers, the percentages of $\mathrm{CD}^{+}$and $\mathrm{CD}^{+} / \mathrm{CD}^{+}$double-positive $\mathrm{T}$ cells, and cross-protection against $S$. suis serotype 1 heterologous challenge [19]. Since weaning piglets are more susceptible to $S$. suis infections due to the stress associated with weaning, prepartum immunization in sows may convey passive immunity to piglets and provide protection. This approach has been proven effective in preventing common swine diseases such as atrophic rhinitis [20], food-and-mouth disease [21] and classical swine fever [22]. Moreover, immunizing pregnant sows with a vaccine containing recombinant Pasteurella multocida toxin (rsPMT) plus Pasteurella multocida type A bacterin as the antigens significantly increased neutralizing antibody titer in colostrum when compared to pregnant sows immunized with the vaccine containing rsPMT only [20]. The combination of recombinant antigens with inactivated bacteria may provide extra antigens and elicit more broaden ranged protection in immunized animals. In the present study, pregnant sows were immunized with the vaccine containing inactivated $S$. suis serotype 2 plus rSao-L as the antigens. Passive immunity in their piglets was analyzed by examining serum antigen-specific antibody titers, levels of various cytokines, including interferon (IFN)- $\gamma$, interleukin (IL)-4, IL-6, IL-8, IL-12, and tumor necrosis factor (TNF)- $\alpha$, and clinical signs after heterologous and homologous challenges with $S$. suis serotype 1 and 2 .

\section{Methods}

\section{Bacterial strains and expression of rSao}

S. suis serotype 1 and 2 strains were obtained from the Pingtung County Animal Disease Control Center, Pingtung, Taiwan. Bacteria were grown in brain-heart infusion (BHI) broth supplemented with $0.5 \%$ yeast extract (Difco Laboratories, Spark, MD, USA) at $37{ }^{\circ} \mathrm{C}$. The rSao was expressed as previously described [19]. The size of PCR product from the $S$. suis strain BCRC 14750 (ATCC 43765) was 2013 bp. Primers for rSao gene were designed according to GenBank accession no. JF 810176 (Sao-F: GCGGGAT CCATGAAT ACTAAGAAATGGAG and Sao-R: CAGAA GCTTGAA CTAATTTACGTTTACGTG). The primers contained restriction enzyme (Bam HI/Hind III) cutting sites and the PCR product was cloned into the expression vector $\mathrm{pET} 32 \mathrm{a}$ 
according to the manufacturer's instructions (Novagen, Darmstadt, Germany). E. coli strain BL21 (DE3) harboring the recombinant plasmid was cultured in Luria-Bertanior modified medium (tryptone: $6 \mathrm{~g} / \mathrm{L}$, soytone: $1.5 \mathrm{~g} / \mathrm{L}$, yeast extract: $7.5 \mathrm{~g} / \mathrm{L}, \mathrm{KH}_{2} \mathrm{PO}_{4:} 1.2 \mathrm{~g} / \mathrm{L}, \mathrm{K}_{2} \mathrm{HPO}_{4}: 0.6 \mathrm{~g} / \mathrm{L}$, glucose: $2 \mathrm{~g} / \mathrm{L})$ at $37{ }^{\circ} \mathrm{C}$ until the absorbance reached 0.6 at $600 \mathrm{~nm}$. Isopropylthio- $\beta$-D-thiogalactose (IPTG) (Amresco, Ohio, USA) was added at a final concentration of $1 \mathrm{mM}$, and the culture was grown for $4 \mathrm{~h}$ with agitation. The resulting culture was ultrafiltrated by Vivaspin 20 100KDa MWCO (GE Healthcare, UK). The concentrated culture was analyzed by SDS-PAGE and Western blotting using the antisera from rabbits immunized with purified rSao. Contamination of endotoxin in the crude rSao was examined using Limulus Amebocyte Lysate test, LAL (CAPE COD, MA, USA) and the result was negative (no clotting, indicating that the concentration of endotoxin is less than $0.125 \mathrm{IU} / \mathrm{mL}$ ).

\section{Purification of rSao}

E. coli expressing rSao were centrifuged at $4{ }^{\circ} \mathrm{C}, 14,000$ $\mathrm{xg}$ for $20 \mathrm{~min}$. The pellet was resuspended in denaturing lysis buffer, sonicated using Misonix Sonicator S-4000 (Misonix, New York, USA), and filtered $(0.22 \mu \mathrm{m})$. The rSao was purified using the ProfiniaTM Protein Purification System (BIO-RAD, California, USA) according to the manufacturer's instructions. Urea was incrementally dialyzed out of the samples, from $6 \mathrm{M}, 5 \mathrm{M}, 4 \mathrm{M}, 3 \mathrm{M}, 2 \mathrm{M}$, and $1 \mathrm{M}$ urea to $0.85 \%$ saline, using $12,000-14,000 \mathrm{MWCO}$ Dialysis Membrane (Cellu•Sep ${ }^{\curvearrowleft}$ MFPI,Orange Scientific, Braine-l'Alleud, Belgium). Thereafter, BSA was used as the standard for determining the concentration of rSao.

\section{Preparation of vaccines}

Formalin-inactivated $S$. suis serotype 2 bacteria and rSao in the aqueous phase final concentration in the vaccine for S. suis and rSao was $1 \times 10^{9} \mathrm{CFU} / \mathrm{mL}$ and $100 \mu \mathrm{g} / \mathrm{mL}$, respectively. The oil vaccine prepared with internal aqueous phase was emulsified with $25 \%$ of water-in-oilin water $(\mathrm{w} / \mathrm{o} / \mathrm{w})$ adjuvant containing two inactivated antigens of $S$. suis and rSao: oil phase: external aqueous phase ratios of 1:1:2 [19,23]. The vaccine did not contain any viable microorganisms, which was confirmed by sterility test.

\section{Immunization of pregnant sows}

Ten healthy pregnant sows, without previous exposure to $S$. suis (S. suis 2 antibody negative), were divided into two groups ( $n=5$ each). The vaccinated group was intramuscularly immunized with $S$. suis serotype 2 plus rSao vaccine $(2 \mathrm{~mL}) 6$ and 2 (boost) weeks before parturition. The other group was injected with an equal volume of sterile saline as the unvaccinated control. Animal experiments were conducted in a private pig farm in Tainan County, Taiwan, with informed consent from the owner to conduct research. The pigs were selected and randomly assigned to the treatment groups for the vaccine trial. All experimental protocols for animal trials were approved by the Animal Care and Use Committee, National Pingtung University of Science and Technology (NPUST). The experiments were conducted based on the Ethical Rules and Law of NPUST.

\section{Assessment of passive immunity in piglets}

Blood samples $(3-4 \mathrm{~mL})$ were collected from the superior vena cava 2,4 , and 6 weeks after parturition from 5 healthy piglets randomly selected from each groups $(n=5)$ for determining the serum antibody titer and cytokine levels. Five weeks after parturition, 3 piglets were randomly selected from each group $(n=3)$ and challenged i.p. with $2 \mathrm{~mL}$ $\left(1.06 \times 10^{9} \mathrm{CFU} / \mathrm{mL}\right)$ of live $S$. suis serotype 1 (P1) or $S$. suis serotype 2 (P2) bacteria. The piglets were monitored daily for clinical signs, body temperature (fever was defined as anal temperature $\geqq 40{ }^{\circ} \mathrm{C}$ ), and bacteremia. Seven days after the challenge, the piglets were i.m. injected with Stresnil $(0.1 \mathrm{~mL} / \mathrm{kg})$, sacrificed, and dissected. Thereafter, pathological examination was performed and lesion score was calculated based on the area of lesions in an organ, where no lesion $=0$, lesion area $<33 \%=1$, lesion area $33-66 \%=3$, lesion area $>66 \%=5$. The necropsy samples of various organs were cultured for bacteriological analysis.

\section{Indirect enzyme-linked immunosorbent assay (ELISA) for serum antibody titers}

Flat-bottomed 96-well plates were coated with either $S$. suis serotype 2 bacteria $(1.25 \mu \mathrm{g} / \mathrm{mL})$ or purified $\mathrm{rSao}$ $(1.25 \mu \mathrm{g} / \mathrm{mL})$ in the coating buffer $\left(15 \mathrm{mM} \mathrm{Na} \mathrm{CO}_{3}\right.$, $35 \mathrm{mM} \mathrm{NaHCO}, 3 \mathrm{mM} \mathrm{NaN}_{3}, \mathrm{pH}=9.6$ ) at $4{ }^{\circ} \mathrm{C}$ overnight. The serum antibody titers were determined as described [19], where $\mathrm{S} / \mathrm{P}$ ratio $=\left(\right.$ Sample $\left.-\mathrm{NC}^{-} \mathrm{x}\right) /$ $\left(\mathrm{PC}^{-} \mathrm{x}-\mathrm{NC}^{-} \mathrm{x}\right)$ (NC: negative control, $\mathrm{PC}$ : positive control). To obtain positive serum, S. suis-negative SPF pigs were challenged with $2 \mathrm{ml}\left(1 \times 10^{9} \mathrm{CFU} / \mathrm{ml}\right)$ S. suis 2 and serum was collected 14 days later.

\section{Detection of serum cytokine levels}

The serum cytokine levels, including IFN- $\gamma$, TNF- $\alpha$, IL4, IL-6, IL-8 and IL-12, were analyzed using the MILLPLEX ${ }^{\oplus}$ MAP Kit (EMD Millipore Corporation, Billerica, MA, USA) according to the manufacturer's instructions. The plates were read by MAGPIX ${ }^{\oplus}$ plate reader at $470-$ 565 nm (EMD Millipore Corporation).

\section{Detection of bacteremia by multiplex PCR}

The detection of bacteremia in blood and organ samples after challenge was carried out using bacteriology and the Multiplex PCR based method. Primers for S. suis cps II and Sao gene were designed by Smith [24] and Hsueh [19], respectively: (cps II-F: GGCGGTCTAGCAGATGC 
Table 1 Fever and bacteremia of piglets after challenge with heterologous Streptococcus suis serotype 1 bacteria

\begin{tabular}{|c|c|c|c|c|c|c|c|c|}
\hline \multirow[t]{2}{*}{ Group $^{a}$} & \multicolumn{8}{|c|}{ Cases of fever/ bacteremia ${ }^{\mathrm{b}}(\%)$} \\
\hline & Day 0 & 1 & 2 & 3 & 4 & 5 & 6 & 7 \\
\hline accination ${ }^{c}$ & $0 / 0$ & $0 / 0$ & $0 / 33$ & $0 / 0$ & $0 / 0$ & $0 / 0$ & $0 / 0$ & $0 / 0$ \\
\hline Control & $0 / 0$ & $33^{d / 100}$ & $33^{e} / 100$ & $0 / 0^{f}$ & $0 / 0$ & $0 / 0$ & $0 / 0$ & م/ \\
\hline \multicolumn{9}{|c|}{ 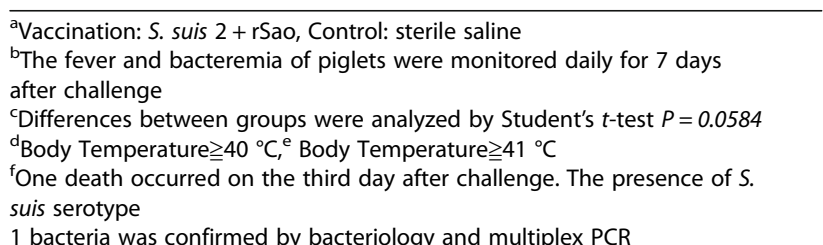 } \\
\hline
\end{tabular}

TCG and cps II-R: GCGAACTGTTAGCAATGAC) (Sao-F: GCGGGATCCATGAATACTAAGAAATGGAG and Sao-R: CAGAAGCTTGAACTAATTTACGTTTAC GTG). Bacterial DNA was isolated using the Blood \& Tissue Genomic DNA extraction Miniprep System kit (Viogene, Sunnyvale, CA, USA). The genes were amplified by 30 PCR cycles consisting of $1 \mathrm{~min}$ denature at $94{ }^{\circ} \mathrm{C}$, 2 min annealing at $56^{\circ} \mathrm{C}$ and 2 min elongation at $72{ }^{\circ} \mathrm{C}$.

\section{Statistical analysis}

All data were expressed as mean \pm S.E.M. and were compared using Student's $t$-test. A $p$-value of $<0.05$ was considered significant.

\section{Results}

\section{Incidence of fever and bacteremia after challenge}

Two piglets from the control group had fever on the first $\left(\geqq 40{ }^{\circ} \mathrm{C}\right)$ and second $\left(\geqq 41{ }^{\circ} \mathrm{C}\right)$ day after challenge. One of the piglets with fever was confirmed to have bacteremia and died on the third day after challenge. In contrast, all piglets from the vaccinated group remained healthy and no fever was observed (Table 1). Results of multiplex PCR demonstrated that all the piglets in the control group had bacteremia during the first two days after challenge (Fig. 1). On the other hand, only one piglet in the vaccination group had bacteremia on the second day (Table 1) and seventh day (Table 2) after challenge. The difference between the vaccinated and the control group was marginal significant $(P=0.058$ and $P=0.140)$ (Tables 1 and 2).

\section{Serum antigen-specific antibody titers}

Results indicated that antigen-specific antibodies, IgG against $S$. suis serotype 2 bacteria and rSao, in serum were significantly $(P<0.05)$ increased in piglets born from vaccinated sows in comparison with those in piglets born from the unvaccinated sows at 2, 4, and 6 weeks after parturition (Fig. 2a and b). The titer of serum IgG against $S$. suis 2 , but not rSao, was remarkably reduced for piglets 6 weeks of age.

\section{Serum cytokine levels}

The concentration of IFN- $\gamma$ in serum was significantly $(P<0.05)$ increased in the piglets from vaccinated sows at 2 and 4 weeks of age when compared to that of piglets from unvaccinated sows (Fig. 3a). Whereas the concentrations of IL-4 (Fig. 3b), IL-6 (Fig. 3c), and IL-12 (Fig. 3d) were significantly $(P<0.05)$ elevated in piglets from vaccinated sows at 2,4 , and 6 weeks after parturition when compared to those of piglets from unvaccinated sows. In contrast, piglets from vaccinated sows had a significantly $(P<0.05)$ higher concentration of IL-8 at 2 , but not 4 and 6 weeks of age (Fig. 3e), when compared to piglets from vaccinated sows. In the case of TNF- $\alpha$, no differences were observed between the two groups at all the time points (Fig. 3f).

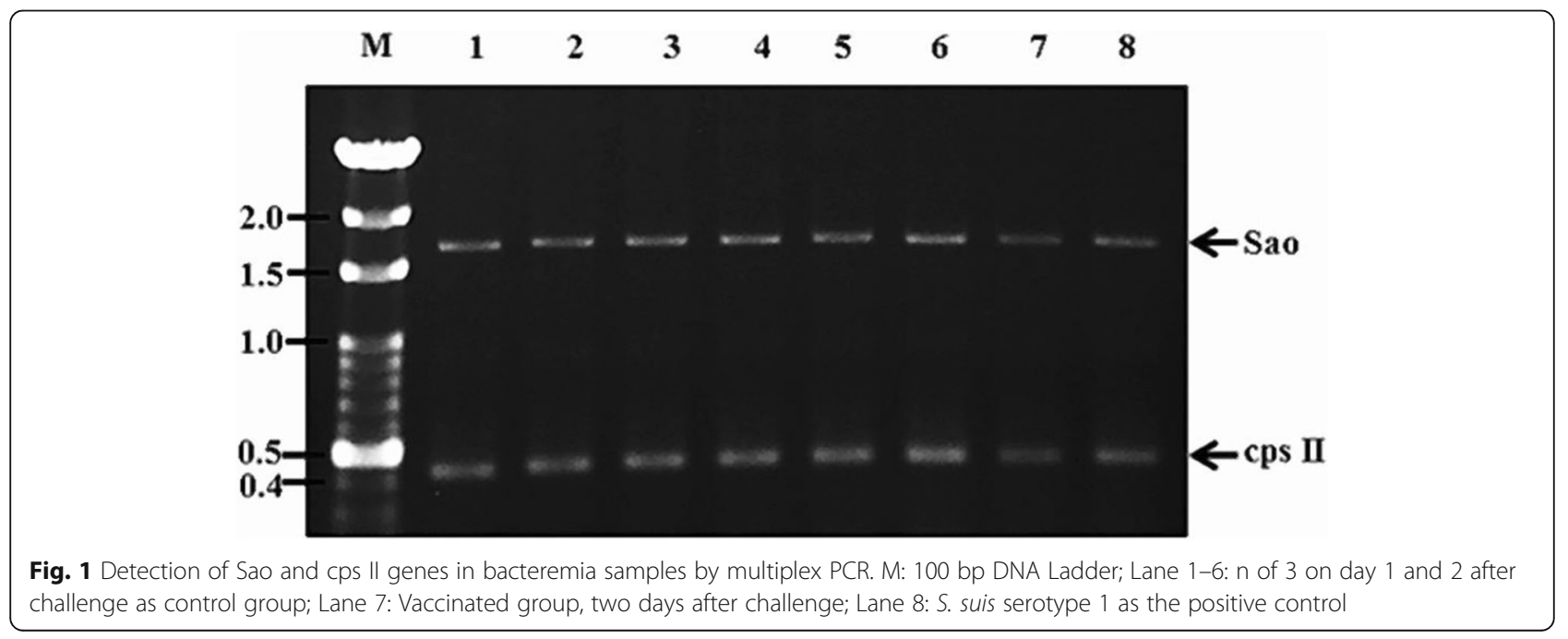


Table 2 Fever and bacteremia of pig after challenge with homologous Streptococcus suis serotype 2 bacteria

\begin{tabular}{lllllllll}
\hline Group $^{\mathrm{a}}$ & \multicolumn{8}{l}{ Cases of fever / bacteremia $^{\mathrm{b}}(\%)$} \\
\cline { 2 - 9 } & Day 0 & 1 & 2 & 3 & 4 & 5 & 6 & 7 \\
\hline Vaccination $^{c}$ & $0 / 0$ & $33 / 0$ & $0 / 0$ & $0 / 0$ & $33 / 0$ & $0 / 0$ & $0 / 0$ & $0 / 0$ \\
Control & $0 / 0$ & $33^{\mathrm{d}} / 0$ & $33 / 0$ & $0 / 0$ & $0 / 0$ & $0 / 0$ & $0 / 0$ & $0 / 33$
\end{tabular}

aaccination: S. suis $2+$ rSao, Control: sterile saline

${ }^{\mathrm{b}}$ The fever and bacteremia of piglets were monitored daily for 7 days after challenge

'Differences between groups were analyzed by Student's $t$-test $P=0.140$

diody Temperature $\geqq 40^{\circ} \mathrm{C}$

\section{Clinical symptoms and pathological lesions after sacrifice}

According to the pathological examination, piglets from sows in the control group showed various clinical signs, including fever, anorexia, and gross lesions in the peritoneal cavity, joints and lungs, in response to the challenge. In contrast, piglets from sows in the vaccinated group remained healthy and only mild lesions were observed. Results from the lesion score indicated that prepartum vaccination in sows significantly $(P<0.05)$ reduced the lesion score by $75 \%$ (7 v.s. 27 ) and $81 \%$ (4 v.s.
21 ) in their piglets after challenge with $S$. suis serotype 1 and 2 bacteria, respectively (Tables 3 and 4). Presence of $S$. suis bacteria in the necropsy samples of various organs (synovial fluid, peritoneal cavity and lungs) was verified and confirmed, using multiplex PCR, to be the $S$. suis serotype 1 and 2 strains used for challenge (Tables 1 and 2).

\section{Histopathological examination}

All piglets survived from challenge were sacrificed and dissected on the $7^{\text {th }}$ day after challenge. Thereafter, histopathological examination was performed. Piglets from unvaccinated sows displayed signs of lesions in the brain and lung, including slight vascular congestion on the brain meninges, influx of neutrophils, lymphocytes, and shedded epithelial cells in the lung alveolar space, and accumulation of neutrophils in the bronchium cavity, indicating the development of bronchopneumonia, hemorrhagic bronchial pneumonia, and lung abscess (Fig. 4a and b). In contrast, above-mentioned signs of

a)

anti- $S$. suis 2
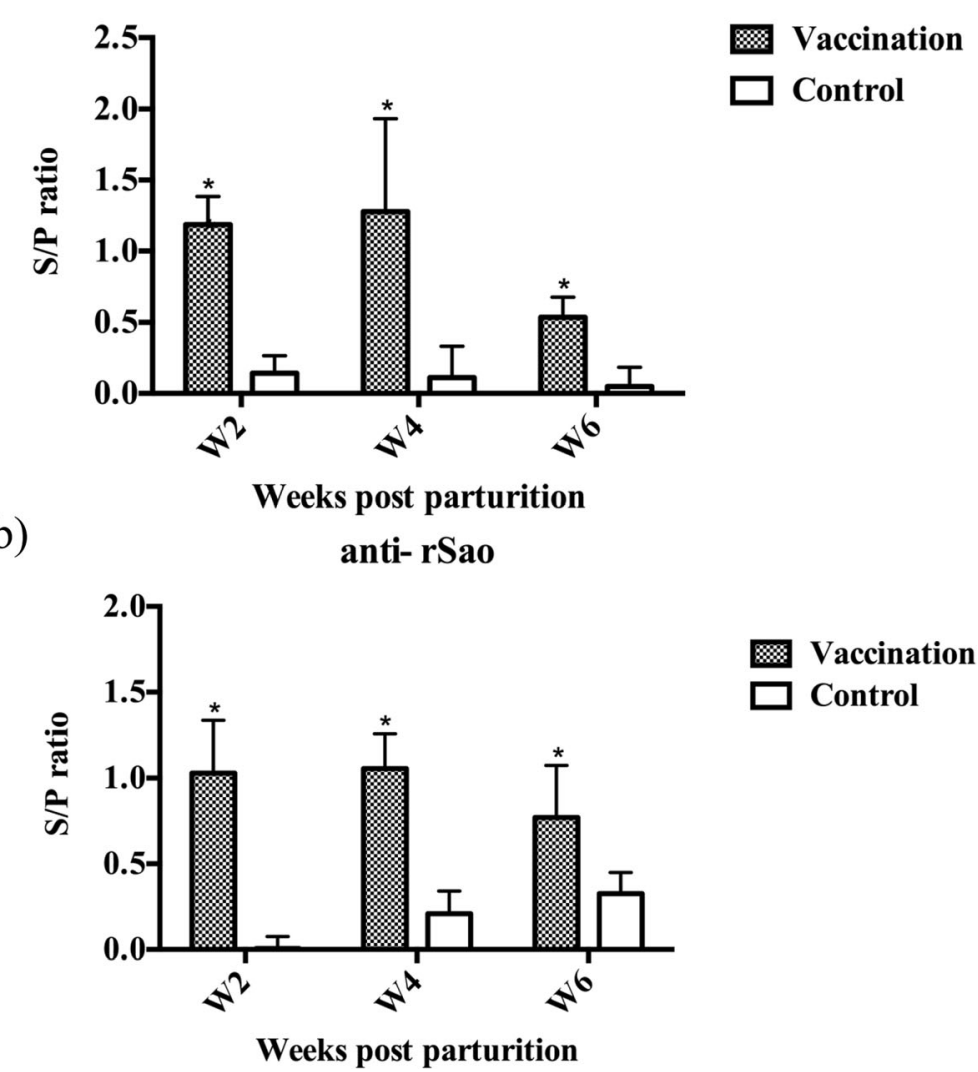

Fig. 2 The antibody response of (a) S. suis 2 (b) rSao-specific IgG in the serum of piglets from sows with or without ( $n=5$ each) prepartum immunization with inactivated S. suis $2+$ rSao. Data are represented as Mean \pm SEM of peak absorbance values as determined by indirect ELISA performed on diluted (1:500) serum samples. S/P ratio $=\left(\right.$ sample $\left.-N^{-} \mathrm{X}\right) /\left(\mathrm{PC}^{-} \mathrm{x}-\mathrm{N} \mathrm{C}^{-} \mathrm{x}\right)$. Differences between groups were analyzed by Student's $t$-test, statistically significant difference $(P<0.05)$ in comparison to the control group 
a)

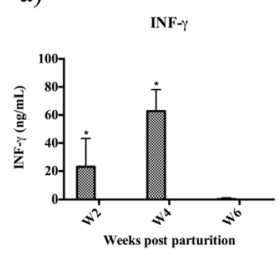

d)

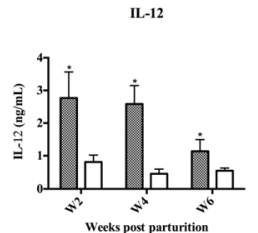

b)

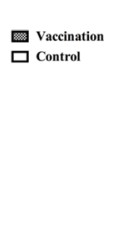

e)

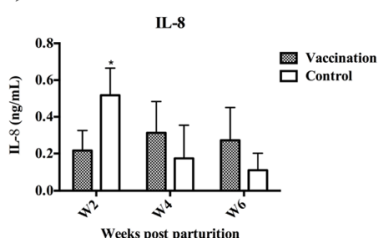

c)

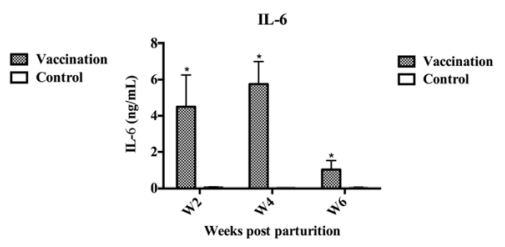

f)

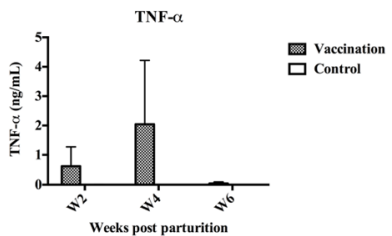

Fig. 3 The concentration of cytokine (a) IFN- $\gamma(\mathbf{b}) I L-4$ (c) IL-6 (d) IL-12 (e) IL-8 (f) TNF-a in piglets from sows with or without ( $n=5$ each) prepartum immunization with inactivated S. suis $2+$ rSao. Data are represented as Mean \pm SEM of the serum concentration of each cytokines determined by MILLPLEX ${ }^{\circledR}$ MAP Kit. Differences between groups were analyzed by Student's $t$-test, statistically significant difference $(P<0.05)$ in comparison to the control group

lesions were not observed in piglets from vaccinated sows after dissection.

\section{Discussion}

Piglets are more susceptible to infections due to the stress associated with weaning and the immature immune system during the early stage of life. Prepartum immunization in sows has been proven safe and efficiently conveys protection to piglets through the transfer of passive immunity in milk. Prepartum vaccination with a subunit vaccine containing recombinant Pasteurella multocida toxin proteins as the antigen in sows was able to enhance the level of neutralizing antibodies, reduce the degree of turbinate atrophy, and increase the survival rate in piglets with experimentally induced progressive atrophic rhinitis (PAR) [20]. Immunizing pregnant sows (52 and 32 days before parturition, respectively) with a type $\mathrm{O} 1$ foot and mouth disease oil emulsion vaccine significantly increased the serum level of neutralizing antibodies in piglets 3 days after birth [21]. Moreover, 3-week-old piglets born from sows immunized with a classical swine fever vaccine (E2) twice after insemination had significantly higher maternal antigen-specific antibodies as indicated by the $\mathrm{ND}_{50}$ (neutralization dilution 50\%) [22].
We previously demonstrated that immunization with the rSao (from serotype $2 \mathrm{~S}$. suis) protein expressed by bioreactors formulated with $\mathrm{w} / \mathrm{o} / \mathrm{w}$ adjuvant increased antigen-specific antibody titers, the percentages of $\mathrm{CD} 8^{+}$ and $\mathrm{CD}^{+} / \mathrm{CD}^{+}$double-positive T cells, and successfully provided cross-serotype protection against the challenge of heterologous S. suis serotype 1 bacteria in pigs [19]. In combination with Quil A, rSao can reduce clinical symptoms after challenge infection with heterologous $S$. suis and stimulates strong opsonizing antibody responses [18]. A previous study has indicated that the pregnant sows immunized with a PAR vaccine containing three short fragments of recombinant subunit Pasteurella multocida toxin (rsPMT) in combination with Pasteurella multocida type A bacterin (rsPMT $+P$. multocida bacterin) as the antigens can elicit higher neutralizing antibody titer in colostrum than pregnant sows immunized with the vaccine containing rsPMT only (1:101 v.s. 1:80) [20]. The immunization protected $60 \%$ of piglets born from these sows after challenged by intramuscular injection with five-fold lethal dose of authentic PMT. The results implied that combination of recombinant antigen with inactivated bacteria as the antigen may enhance the immune responses elicited by the vaccine. In

Table 3 Percentage of pathological lesion score after challenge with heterologous Streptococcus suis serotype 1 bacteria

\begin{tabular}{|c|c|c|c|c|c|c|c|c|}
\hline \multirow[t]{2}{*}{$\overline{\text { Group }^{a}}$} & \multirow{2}{*}{$\begin{array}{l}\text { Total } \\
\text { Score }\end{array}$} & \multirow{2}{*}{$\begin{array}{l}\text { Reduced } \\
\text { Percentage }\end{array}$} & \multicolumn{6}{|c|}{ The Score of Gross Lesions ${ }^{\mathrm{b}}$} \\
\hline & & & Brain & Serosa & Joint & Spleen & Liver & Lung \\
\hline Vaccination & 7 & $75 \%^{c}$ & $1(1 / 3)$ & $0(0 / 3)$ & $3(1 / 3)$ & $0(0 / 3)$ & $0(0 / 3)$ & $3(1 / 3)$ \\
\hline Control $^{d}$ & 27 & - & $1(1 / 3)$ & $7(3 / 3)$ & $7(3 / 3)$ & $1(1 / 3)$ & $4(2 / 3)$ & $7(3 / 3)$ \\
\hline
\end{tabular}

Vaccination: $S$. suis $2+$ rSao, Control: sterile saline

${ }^{\mathrm{b}}$ No lesion $=0$, lesion area $<33 \%=1$, lesion area $33-66 \%=3$, lesion area $>66 \%=5$

'Differences between groups were analyzed by Student's $t$-test $P<0.05$

${ }^{\mathrm{d}}$ The number of piglets in a group that was confirmed to have the presence of $S$

suis serotype 1 bacteria 
Table 4 Percentage of pathological lesion score after challenge with homologous Streptococcus suis serotype 2 bacteria

\begin{tabular}{|c|c|c|c|c|c|c|c|c|}
\hline \multirow[t]{2}{*}{ Group $^{a}$} & \multirow{2}{*}{$\begin{array}{l}\text { Total } \\
\text { Score }\end{array}$} & \multirow{2}{*}{$\begin{array}{l}\text { Reduced } \\
\text { Percentage }\end{array}$} & \multicolumn{6}{|c|}{ The Score of Gross Lesions ${ }^{b}$} \\
\hline & & & Brain & Serosa & Joint & Spleen & Liver & Lung \\
\hline Vaccination & 4 & $81.0 \%{ }^{\mathrm{e}}$ & $0(0 / 3)$ & $0(0 / 3)$ & $0(0 / 3)$ & $0(0 / 3)$ & $0(0 / 3)$ & $4(2 / 3)$ \\
\hline Control $^{d}$ & 21 & - & $0(0 / 3)$ & $0(0 / 3)$ & $0(0 / 3)$ & $5(3 / 3)$ & $3(3 / 3)$ & $13(3 / 3$ \\
\hline
\end{tabular}

Vaccination: S. suis2 + rSao, Control: sterile saline

${ }^{b}$ No lesion $=0$, lesion area $<33 \%=1$, lesion area $33-66 \%=3$, lesion area $>66 \%=5$

'Differences between groups were analyzed by Student's $t$-test $P<0.05$

${ }^{\mathrm{d}}$ The number of piglets in a group that was confirmed to have the presence of $S$ suis serotype 2 bacteria

addition, virulence factors of S. suis other than Sao, such as suilysin, muramidase-released protein (MRP), and extracellular factor $(\mathrm{EF})$, also play a role in the pathogenesis of S. suis. Immunization with a subunit vaccine containing these virulence factors $\left(\mathrm{MRP}^{+} / \mathrm{EF}^{+}\right)$from $S$. suis serotype 2 as the antigens has been demonstrated to protect pigs from challenge of homologous or heterologous S. suis strains [25]. Addition of inactivated S. suis bacteria containing these surface virulent factors into the vaccine formula may provide extra antigens and elicit broader protection in immunized animals. Therefore, pregnant sows were immunized with the vaccine containing inactivated $S$. suis serotype 2 plus rSao as the antigens and the passive immunity against heterologous S. suis serotype 1 infection in their piglets was investigated in the present study.

The serum titer of IgG against rSao and S. suis serotype 2 in piglets from vaccinated sows remained significantly higher than that of piglets from unvaccinated sow until 6 weeks of age (Fig. 2a and b). After challenge with live $S$. suis serotype 1 bacteria, the lesion score of piglets from vaccinated sows was reduced by $75 \%$. These results implied that immunization with rSao plus inactivated $S$. suis serotype 2 in pregnant sows enhanced the passive immunity against $S$. suis and provided cross-protection in their piglets. The Sao protein is highly conserved among $S$. suis strains and Sao-specific antibodies have been shown to cross-react with 28 different serotypes of S. suis [13]. The lesion score was reduced by $60 \%$ in pigs immunization with rSao alone after challenge with live heterologous S. suis bacteria [19]. Results from the present study revealed that the lesion score was reduced by $75 \%$ in piglets born from sows immunized with inactivated S. suis bacteria plus rSao after challenge with live heterologous S. suis bacteria.

In piglets produced by vaccinated sows, the antigenspecific IgG titer remained high until 6 weeks of age (Fig. 2a and b). This is in agreement with a previous study in which pregnant sows were immunized with the autogenous $S$. suis bacterin and MRP-specific IgG remained detectable until 6 weeks of age [25]. However, a remarkable reduction of the antigen-specific IgG titer in serum was observed in the 6 weeks of age in comparison with the titers in the 2 and 4 weeks of age, which is possibly due to the half-life of IgG absorbed from colostrum. The IgG concentrations in piglets at $24 \mathrm{~h}$ of age

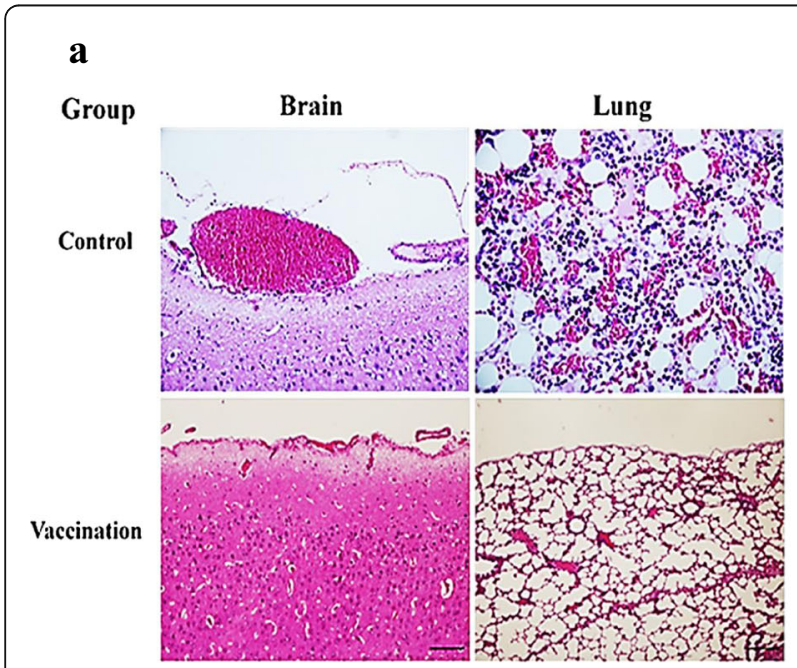

\section{b}

Group (Lung) Gross Lesion

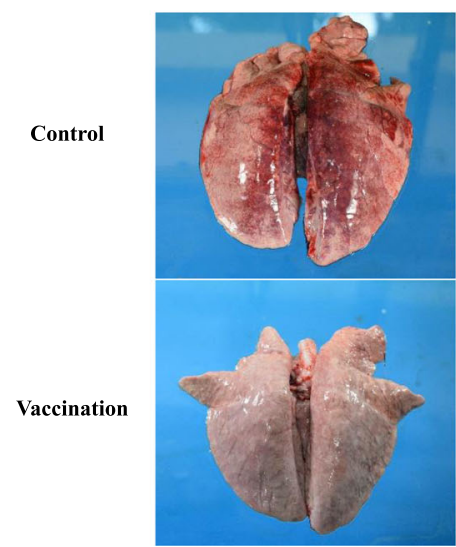

Histopathological

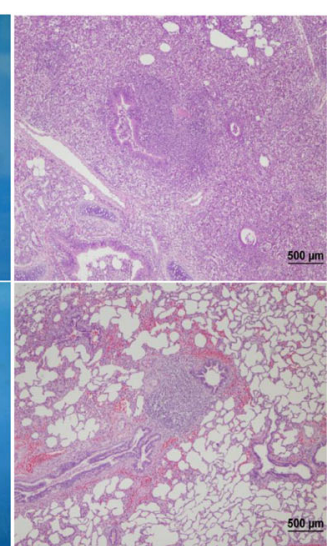

Fig. 4 Results of histopathological examination. Piglets from sows with or without prepartum immunization with inactivated S. suis $2+$ rSao were challenged with (a) heterologous S. sui serotype 1 and (b) homologous S. sui serotype 2 bacteria at the 5 weeks of age. The histopathological examination was performed on the $7^{\text {th }}$ day after the challenge. Control pigs manifested severe brain and lung lesions, including slight vascular congestion on the brain meninges and shedded epithelial cells in the lung alveolar space 
ranged from 18.7 to $39 \mathrm{mg} / \mathrm{mL}$ and gradually decreased to $6.3 \mathrm{mg} / \mathrm{mL}$ at 5 to 6 weeks of age [26]. The w/o/w adjuvant used in our vaccine has been shown to stimulate both humoral and cell-mediated arms of immune responses [17, 23].

Compared to piglets from sows in the control group, piglets from sows in the vaccinated group had significantly $(P<0.05)$ increased levels of IL-4, IL-6, IL-12, and IFN- $\gamma$ in serum after parturition. The source of these cytokines in piglets' circulation could be mainly from the absorption of cytokines in colostrum before gut closure. IL-4 and IL-6 plays a role in enhancing Th2-type immune responses, B cell proliferation, and antibody production [27]. On the other hand, IL-12 and IFN- $\gamma$ are the key cytokines that lead immune responses to Th1-type. IL-12 activates immune cells, such as NK cells and naïve $\mathrm{CD} 4^{+} \mathrm{T}$ cells, to secret IFN- $\gamma$, which in turn facilitate macrophages maturation [28-32]. Therefore, the higher concentration of cytokines in serum may contribute, at least partially, to the enhanced resistance to challenge with live S. suis bacteria in piglets from vaccinated sows. It is unclear why the level of serum IL-8 was increased only at 2 weeks after parturition in piglets from sows in the control group. IL-8 is a potential chemoattractant that is up regulated during the course of intramammary infections [33]. However, whether the sows had mastitis was not determined in our study. The serum levels of TNF- $\alpha$ were low and not different between the two groups, which is probably due to the short half-life of this cytokine [27].

Results of the histopathological examination revealed that piglets from unvaccinated sows had slight vascular congestion on the brain meninges, influx of neutrophils, lymphocytes, and shedded epithelial cells in the lung alveolar space, and signs of suppurative bronchopneumonia, hemorrhagic bronchial pneumonia, and lung abscess (Fig. 4a and b). In piglets i.v. challenged by live S. suis, the main lesions (found in the joints, spleen, liver, and kidneys) are associated with synovitis, spleen inflammation, hepatitis, and pneumonia [25]. Dissection of the piglet that died on the third day after challenge showed severe lesions in the lung. In contrast, lesions were not found in any of the piglets from sows in the vaccinated group after dissection, indicating that the enhanced passive immunity against $S$. suis was sufficient to protect piglets from $S$. suis challenge.

\section{Conclusions}

Prepartum immunization of inactivated $S$. suis bacterin plus rSao in sows was able to increase serum antigenspecific antibody titers, the concentrations of IFN- $\gamma$, IL4, IL-6 and IL-12 in serum, and provide cross-protection against $S$. suis infections in the piglets. The protection was characterized by the reduction on lesion score $(75$ and $81 \%$ ), bacteremia, and fever. This vaccine and immunization approach may be applied to enhance the protection against both heterologous and homologous $S$. suis infections in newborn piglets.

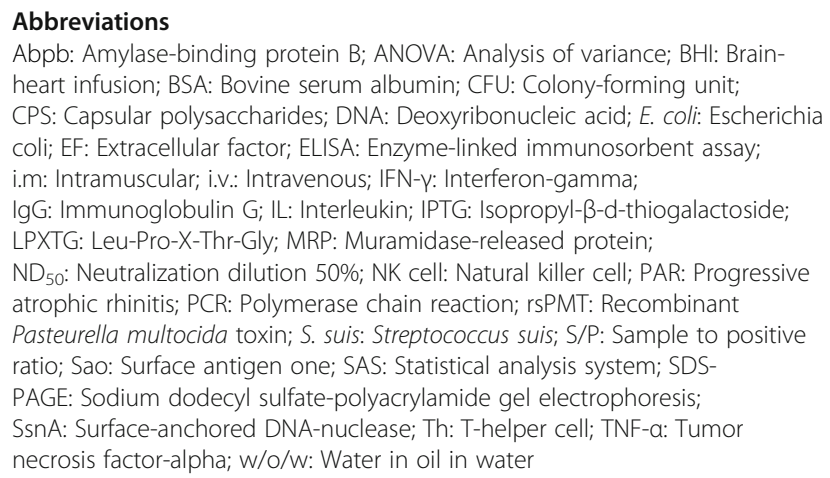

\section{Acknowledgements}

We thank Dr. Tsung-Chou Chang of the Department of Veterinary Medicine, NPUST, for pathological evaluation of the pigs.

\section{Funding}

This study was partially supported by the grant from the National Science Council, Taiwan (NSC 99-2324-B-020-001-MY2).

\section{Availability of data and materials}

All data were presented in the manuscript.

\section{Authors' contributions}

$\mathrm{KHH}$ performed the laboratory work, participated in data analysis and wrote the main parts of the manuscript. WBC and CYC designed the concept of the study, supervised the experiments, revised and submitted the final manuscript. The animal model was performed by YCC. LTC and JWL performed statistical analysis and prepared the final draft of the manuscript. All authors read and approved the final manuscript.

\section{Competing interests}

All authors have no financial or personal relationships with other people or organizations that could inappropriately influence or bias their work.

\section{Consent for publication}

Not applicable.

\section{Ethics approval}

Animal experiments were conducted in a private pig farm in Tainan County, Taiwan, with informed consent from the owner to conduct research. The pigs were selected and randomly assigned to the treatment groups for the vaccine trial. All experimental protocols for animal trials were approved by the Animal Care and Use Committee, National Pingtung University of Science and Technology (NPUST). The experiments were conducted based on the Ethical Rules and Law of NPUST.

\section{Author details \\ 'Department of Veterinary medicine, College of Veterinary Medicine, National Pingtung University of Science and Technology, Pingtung 91201, Taiwan. ${ }^{2}$ Graduate Institute of Animal Vaccine Technology, College of Veterinary Medicine, National Pingtung University of Science and Technology, 1, Shuehfu Road, Neipu, Pingtung 91201, Taiwan. ${ }^{3}$ Department of Tropical Agriculture and International Cooperation, National Pingtung University of Science and Technology, Pingtung 91201, Taiwan.}

Received: 28 July 2015 Accepted: 22 December 2016 Published online: 07 January 2017

\section{References}

1. Lun ZR, Wang QP, Chen XG, Li AX, Zhu XQ. Streptococcus suis: an emerging zoonotic pathogen. Lancet Infect Dis. 2007;7(3):201-9. 
2. Hill JE, Gottschalk M, Brousseau R, Harel J, Hemmingsen SM, Goh SH. Biochemical analysis, cpn60 and 16S rDNA sequence data indicate that Streptococcus suis serotypes 32 and 34 , isolated from pigs, are Streptococcus orisratti. Vet Microbiol. 2005;107(1-2):63-9.

3. Higgins R, Gottschalk M. Distribution of Streptococcus suis capsular types in 1998. Can Vet J. 1999:40(4):277.

4. Chang CJ, Pan CH, Lee SH, Huang TS, Wang C, Jong MH. Analysis of porcine common pathogens in pig herd in Taiwan. Anim Health Res Inst. 2007;42:71-8.

5. Chu CY, Shu SF, Huang JH, Hsu JP, Xue KR. Rapid detection of Streptococcus suis serotypes and virulent factors in southern Taiwan by multiplex polymerase chain reaction. Taiwan Vet J. 2009;35(2):107-14.

6. Pallares FJ, Schmitt CS, Roth JA, Evans RB, Kinyon JM, Halbur PG. Evaluation of a ceftiofur-washed whole cell Streptococcus suis bacterin in pigs. Can J Vet Res. 2004;68(3):236-40.

7. Wisselink HJ, Stockhofe-Zurwieden N, Hilgers LA, Smith HE. Assessment of protective efficacy of live and killed vaccines based on a non-encapsulated mutant of Streptococcus suis serotype 2. Vet Microbiol. 2002;84(1-2):155-68

8. Elliott SD, Clifton-Hadley F, Tai J. Streptococcal infection in young pigs. V. An immunogenic polysaccharide from Streptococcus suis type 2 with particular reference to vaccination against streptococcal meningitis in pigs. J Hyg (Lond). 1980;85(2):275-85.

9. Goyette-Desjardins G, Calzas C, Shiao TC, Neubauer A, Kempker J, Roy R, et al. Protection against Streptococcus suis Serotype 2 infection using a capsular Polysaccharide Glycoconjugate vaccine. Infect Immun. 2016;84(7):2059-75.

10. Calzas C, Lemire P, Auray G, Gerdts V, Gottschalk M, Segura M. Antibody response specific to the capsular polysaccharide is impaired in Streptococcus suis serotype 2-infected animals. Infect Immun. 2015;83(1):441-53.

11. Okwumabua $\mathrm{O}$, Abdelmagid $\mathrm{O}$, Chengappa MM. Hybridization analysis of the gene encoding a hemolysin (suilysin) of Streptococcus suis type 2: evidence for the absence of the gene in some isolates. FEMS Microbiol Lett. 1999;181(1):113-21.

12. Maione D, Margarit I, Rinaudo CD, Masignani V, Mora M, Scarselli M, et al. Identification of a universal group B streptococcus vaccine by multiple genome screen. Science. 2005;309(5731):148-50.

13. Li Y, Martinez G, Gottschalk M, Lacouture S, Willson P, Dubreuil JD, et al. Identification of a surface protein of Streptococcus suis and evaluation of its immunogenic and protective capacity in pigs. Infect Immun. 2006;74(1):305-12.

14. Feng Y, Pan X, Sun W, Wang C, Zhang H, Li X, et al. Streptococcus suis enolase functions as a protective antigen displayed on the bacterial cell surface. J Infect Dis. 2009:200(10):1583-92.

15. Gomez-Gascon L, Cardoso-Toset F, Amarilla PS, Tarradas C, Carrasco L, Olaya-Abril A, et al. A new recombinant SsnA protein combined with aluminum hydroxide protects mouse against Streptococcus suis. Vaccine. 2014;32(51):6992-9.

16. Huang K, Yuan Z, Li J, Zhang Q, Xu Z, Yan S, et al. Identification and characterisation a surface-associated arginine peptidase in Streptococcus suis serotype 2. Microbiol Res. 2015;170:168-76.

17. Zhang YM, Shao ZQ, Wang J, Wang L, Li X, Wang C, et al. Prevalent distribution and conservation of Streptococcus suis Lmb protein and its protective capacity against the Chinese highly virulent strain infection. Microbiol Res. 2014;169(5-6):395-401.

18. Li Y, Gottschalk M, Esgleas M, Lacouture S, Dubreuil JD, Willson P, et al. Immunization with recombinant Sao protein confers protection against Streptococcus suis infection. Clin Vaccine Immunol. 2007;14(8):937-43.

19. Hsueh KJ, Lee JW, Hou SM, Chen HS, Chang TC, Chu CY. Evaluation on a Streptococcus suis vaccine using recombinant sao-I protein manufactured by bioreactors as the antigen in pigs. Transbound Emerg Dis. 2014;61(6):e35-43.

20. Liao CM, Huang C, Hsuan SL, Chen ZW, Lee WC, Liu Cl, et al. Immunogenicity and efficacy of three recombinant subunit Pasteurella multocida toxin vaccines against progressive atrophic rhinitis in pigs. Vaccine. 2006;24(1):27-35.

21. Francis MJ, Black $L$. The effect of vaccination regimen on the transfer of foot and mouth disease antibodies from the sow to her piglets. J Hyg (Lond). 1984;93(1):123-31.

22. Lipowski A, Drexler C, Pejsak Z. Safety and efficacy of a classical swine fever subunit vaccine in pregnant sows and their offspring. Vet Microbiol. 2000; 77(1-2):99-108.

23. Chu CY, Chen JJ, Chen H. Effect of water-in-oil in-water (W/O/W) oil adjuvant on long-term antibody response of bovine ephemeral fever vaccine Taiwan. Taiwan Vet J. 2007:33(3-4):211-6.
24. Smith HE, Veenbergen V, van der Velde J, Damman M, Wisselink HJ, Smits MA. The cps genes of Streptococcus suis serotypes 1,2, and 9: development of rapid serotype-specific PCR assays. J Clin Microbiol. 1999;37(10):3146-52.

25. Wisselink HJ, Vecht U, Stockhofe-Zurwieden N, Smith HE. Protection of pigs against challenge with virulent Streptococcus suis serotype 2 strains by a muramidase-released protein and extracellular factor vaccine. Vet Rec. 2001; 148(15):473-7.

26. Bourne FJ. The immunoglobulin system of the suckling pig. Proc Nutr Soc. 1973;32(3):205-15.

27. Nguyen TV, Yuan L, Azevedo MS, Jeong KI, Gonzalez AM, Saif LJ. Transfer of maternal cytokines to suckling piglets: in vivo and in vitro models with implications for immunomodulation of neonatal immunity. Vet Immunol Immunopathol. 2007;117(3-4):236-48.

28. Zeng R, Spolski R, Finkelstein SE, Oh S, Kovanen PE, Hinrichs CS, et al. Synergy of $\mathrm{IL}-21$ and $\mathrm{LL}-15$ in regulating $\mathrm{CD} 8+\mathrm{T}$ cell expansion and function. J Exp Med. 2005;201(1):139-48.

29. Arulanandam BP, Lynch JM, Briles DE, Hollingshead S, Metzger DW. Intranasal vaccination with pneumococcal surface protein A and interleukin-12 augments antibody-mediated opsonization and protective immunity against Streptococcus pneumoniae infection. Infect Immun. 2001;69(11):6718-24.

30. Sullivan SP, Koutsonanos DG, Del Pilar MM, Lee JW, Zarnitsyn V, Choi SO, et al. Dissolving polymer microneedle patches for influenza vaccination. Nat Med. 2010;16(8):915-20.

31. Hsieh CS, Macatonia SE, Tripp CS, Wolf SF, O'Garra A, Murphy KM. Development of TH1 CD4+ T cells through IL-12 produced by Listeriainduced macrophages. Science. 1993:260(5107):547-9.

32. Nathan CF, Murray HW, Wiebe ME, Rubin BY. Identification of interferongamma as the lymphokine that activates human macrophage oxidative metabolism and antimicrobial activity. J Exp Med. 1983;158(3):670-89.

33. Zhu Y, Berg M, Fossum C, Magnusson U. Proinflammatory cytokine mRNA expression in mammary tissue of sows following intramammary inoculation with Escherichia coli. Vet Immunol Immunopathol. 2007;116(1-2):98-103.

\section{Submit your next manuscript to BioMed Central and we will help you at every step:}

- We accept pre-submission inquiries

- Our selector tool helps you to find the most relevant journal

- We provide round the clock customer support

- Convenient online submission

- Thorough peer review

- Inclusion in PubMed and all major indexing services

- Maximum visibility for your research

Submit your manuscript at www.biomedcentral.com/submit 